\title{
A Case Study on Frequency Reuse in OFDMA Systems Using Hierarchical Radio Resource Management
}

\author{
Banani Roy ${ }^{1}$, Chanchal Kumar Roy ${ }^{1}$ and Michael Einhaus ${ }^{2}$ \\ ${ }^{1}$ School of Computing, Queen's University, Kingston, ON, Canada K7L 3N6, \\ ${ }^{2}$ Communication Networks, RWTH Aachen University, Aachen, Germany 52074
}

Tel: +1 6135336000 ext: 75103, Fax: +1 613 5336513, E-mail: broy@cs.queensu.ca

\begin{abstract}
This paper presents a case study on the frequency reuse using Hierarchical Radio Resource Management (HRRM) in an OFDMA system. In this HRRM, an Access Point Controller is developed to dynamically assign subchannels to the Access Points (APs) on the basis of interference measurements and traffic situation, and the APs allocate resource elements to the Mobile Terminals (MTs) using an OFDMA scheduler. Using this HRRM we focus on the effect of frequency reuse in the considered system. Three types of reuse constraints with different scaling of distances between the APs are used to analyze frequency reuse effect.
\end{abstract}

Index Terms - OFDMA, Frequency Reuse, Reuse Constraints, Utilization, HRRM.

\section{INTRODUCTION}

In this paper a Hierarchical Radio Resource Management (HRRM) scheme has been developed to investigate the frequency reuse in an Orthogonal Frequency Division Multiple Access (OFDMA) system. In HRRM, resource allocation is done in two steps. First, the Access Point Controller (APC) (centralized allocator) takes the high level resource allocation decision. Then the APC passes the allocation request to the Access Point (AP) (secondary allocator) that takes the detailed resource allocation decision. The dynamic resource allocation on the APC level can be considered as a slow adaptation to the traffic load and interference whereby the resource allocation on the AP level is done in a fast adaptation (frame-by-frame) manner to react on the changing channel conditions (e.g. fading). The HRRM can play a good role in case of radio resource allocation as the complexity of optimizing resource allocation is always a NPhard problem where execution time of the algorithm increases exponentially $[1,10]$.

Frequency reuse implies that in a coverage area there are several cells that use the same set of frequencies due to the propagation path loss of the radio signal [2]. These cells are called co-channel cells, and the interference between signals from these cells is called co-channel interference. The adaptive reuse decision between two APs is taken on the basis of the position of the APs and its associated MTs. If an AP and its associated MTs are spatially apart from the interferer cell (other AP and its associated MTs), then flexible Reuse Constraints (RCs) can be used so that the AP can reuse its interferer AP's resources. On the other hand if the APs are situated closely, strict RCs can be used. An intelligent HRRM with effective frequency reuse can improve the performance of an OFDMA system. The motivation of this paper is to show the effect and importance of frequency reuse in an OFDMA system using different RCs. In this regard a low complexity dynamic subchannel algorithm and an OFDMA scheduler are used from [8] to do the comparative study among different RCs.

\section{HIERARCHICAL RADIO RESOURCE ALLOCATION}

The working process of the APC is synchronized with the MAC frames and it updates subchannels for the APs in some defined frames. The frame level interaction diagram is shown in Fig. 1. In this figure it is shown that the APC updates the resources of APs in the super frame level. The term super frame indicates that the resources of APs are updated by the APC in a frame interval but not at each frame. Here the APC uses a low complexity traffic load adaptive and interference aware subchannel allocation scheme and the AP uses an OFDMA scheduler to assign resource elements to the MTs.

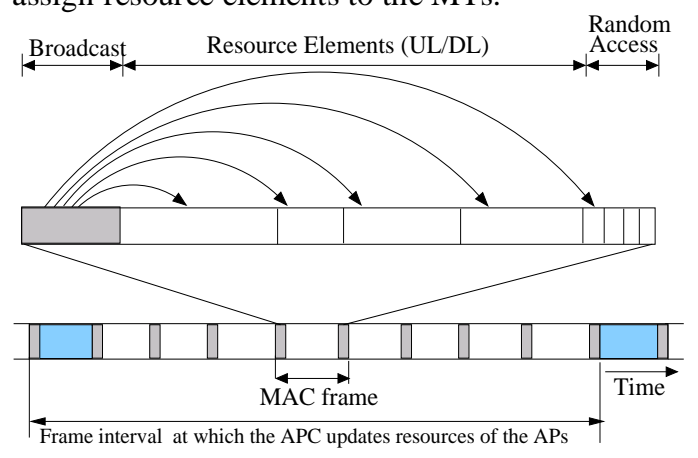

Fig. 1. Frame level interaction between APC \& AP 
The dynamic subchannel allocation algorithm and the OFDMA scheduler are briefly described as follows (for details see [8]):

\section{A. Interference Aware DCA scheme: APC level}

In this interference aware DCA scheme the APC uses two phases: reuse decision phase and allocation phase. In the reuse decision phase the APC decides which AP can reuse which AP's resources in an intelligent manner in order to reduce the co-channel interference. After getting the invocation from the APC, the APs measure the interference using the concept of Self Organizing Reuse Partitioning (SORP )[4] during the broadcast and then get back the measured interference values to the APC. Using those measured values the APC estimates the mutual Signal to Interference Ratio (SIR) for each of the APs. Then it forms co-channel cells by comparing the mutual SIR obtained by applying a RC with a predetermined reuse threshold. In order to investigate the effect of frequency reuse, we have identified three types of frequency RCs; they are as follows:

(a) Minimum ( $\min$ ) RC: Using this constraint the APC estimates the minimum mutual SIR for all the MTs associated to an AP. Here it is considered that two APs can reuse resources, if minimum mutual SIR values of both APs for their associated MTs are greater than or equal to the predefined SIR threshold i.e. position of the single MT restricts the reuse decision.

(b) Mean RC: In this constraint, formation of cochannel cells in APs does not depend on the position of a MT rather this constraint considers position of all the associated MTs of an AP. If the average mutual SIR values of two APs exceed the reuse threshold, they can use the same resources.

(c) Weighted RC: To reduce the bad impact of the min (very strict) and mean (very flexible) $\mathrm{RCs}$, another $\mathrm{RC}$ is introduced. In this case a weighted value is multiplied with mutual SIR value obtained by applying the mean RC. The weighted value is calculated considering the utilization of the interferer AP which is (1utilization). If the utilization of the interferer AP is low then other AP is capable of reusing its (interferer AP) resources since low utilization indicates that interferer $\mathrm{AP}$ is not transferring data to all of its associated MTs.

\section{B. OFDMA Scheduler: AP level}

The scheduling of data packets in OFDMA systems is a challenging task. It is required that the scheduler is able to exploit the multi-user diversity and provides fairness at the same time. A simple adaptive modulation and power allocation scheme is deployed in the used scheduling algorithm. To each allocated subchannel within the same scheduling, an interval (i.e. a time slot) is assigned to the same fraction of the total transmission power. This power allocation scheme does not take into account the current channel characteristics. Therefore, it differs from the water-filling solution which is optimal from the information theoretic point of view [3]. After the power allocation, reasonable modulation schemes are assigned based on Signal to Noise Ratio (SNR) estimations.

\section{SIMULATION ENVIRONMENT AND RESULTS}

The simulation of this work is performed by extending NS2 [7] and the statistical evaluation is conducted following [9]. For the traffic source model, a two-state Markov Modulated Poisson Process (MMPP) [6] is used. Brownian motion mobility [5] is used to implement the mobility of the MTs. In the following a comparative study is made to show the effect of frequency reuse by using the obtained simulation results.

\section{A. Comparison between different RCs}

In this scenario there are five APs: AP0, AP1, AP2, AP3 and AP4 are associated to the APC where each AP has 10 associated MTs. Different simulations are done by varying the load of AP2 and AP4 while keeping fixed the load of AP0, AP1 and AP3 in order to create irregularity in the traffic situation. Two scaling values $1 \& 2$ are used and these values are multiplied to the position of the APs while keeping fixed the mobility region radius, e.g. the scenario shown in Fig. 2 uses scaling value 1 and scenario shown in Fig. 3 uses scaling value 2 .

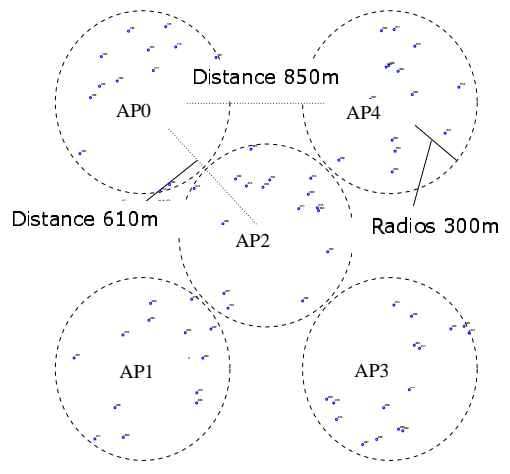

Fig. 2. Five cell scenario with scaling value 1

The mean Packet Error Rate (PER) and mean downlink delay of the whole scenario are shown in Fig. 4(a) and 4(b) respectively for the scenario with scaling value 1 . The curves show that in this 
scenario the mean delay in case of min $\mathrm{RC}$ is the lowest as the PER for the mean RC and the weighted $\mathrm{RC}$ are remarkably higher than min $\mathrm{RC}$ which causes retransmission of packets and increases mean delay.

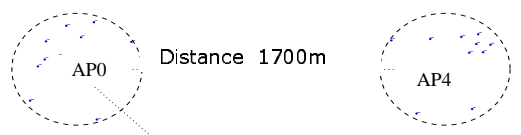

Distance $1220 \mathrm{~m}$
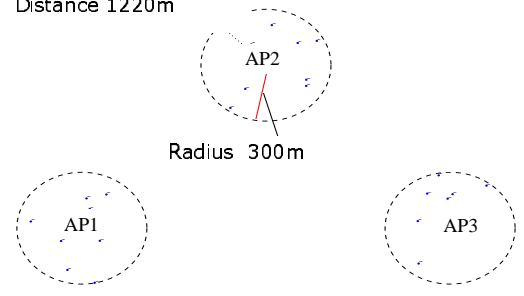

Fig. 3. Five cell scenario with scaling value 2

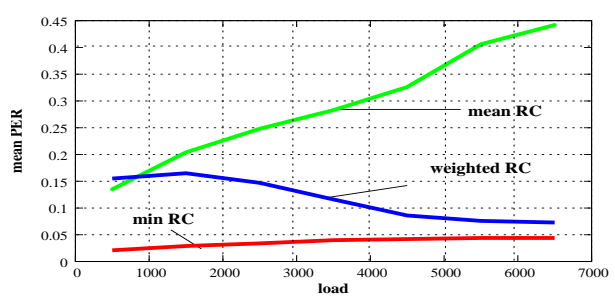

(a) Mean PER for scaling 1

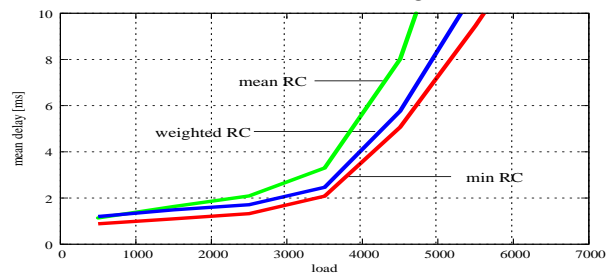

(b) Mean delay for scaling 1

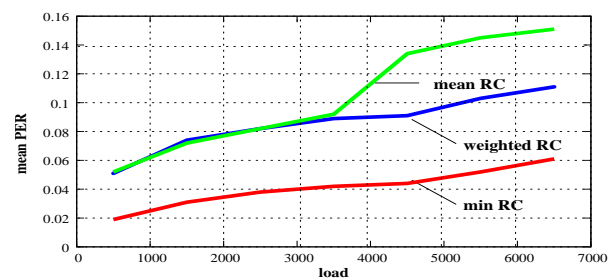

(c) Mean PER for scaling 2

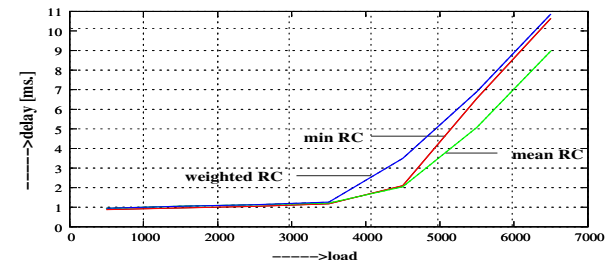

(d) Mean delay for scaling 2

Fig. 4. Comparison among RCs using different scaling value

But when the scaling value 2 is used (see Fig. 3), the mean RC is working better than the min RC. As APs are spatially separated, the co-channel interference (PER is reduced shown in Fig. 4(c) ) is lower and as a result in case of mean $\mathrm{RC}$, the mean delay is lower than that of the other RCs due to the higher reuse of subchannel(s) (see Fig. $4(d))$.

The weighted RC performs better than mean $\mathrm{RC}$ in case of scaling value 1 , because in that case, co-channel interference is reduced for the limited reuse of resources between the APs by multiplying (1- utilization) value with the mean mutual SIR value of each AP at the high load situation. Here at the high traffic load the value (1-utilzation) is low as the utilization of the APs is high.

\section{B. Effect of Reuse using $\min R C$}

While maintaining the inter-cell interference, reuse of subchannels always reduces the mean delay. To show this effect, we have done different simulations with different scaling of distances between the APs based on the scenario shown in Fig. 2. The mean subchannel assignment curves with reuse and without reuse (with reuse: APs can reuse subchannels of other APs, without reuse: APs cannot reuse subchannels of other APs) are shown in Fig. 5(a). The curves show that the average subchannel difference between with reuse and without reuse is less than 0.05. But when scaling value 2 is used, this difference is greater than 5.5 shown in Fig. 5(b). Therefore, in this case the mean delay with reuse is much lower than that of without reuse as shown in Fig.6. The curves show that when scaling value 1 is used, the mean delay curve does not differ a lot as in this case there is less reuse of subchannels which will be explained below.

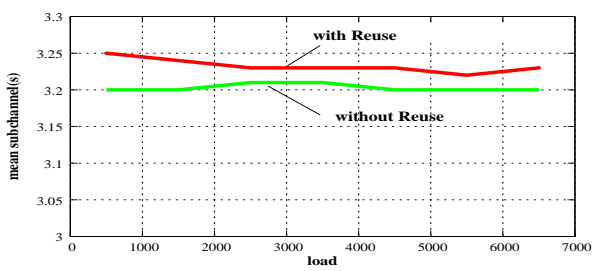

(a) Mean subchannel using scaling value 1

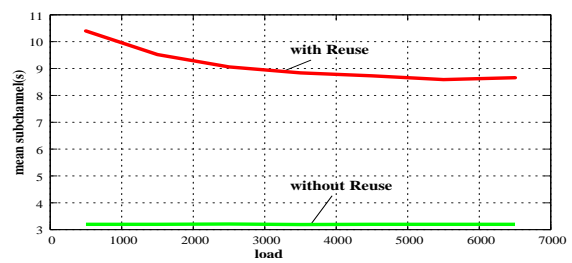

(b) Mean subchannel using scaling value 2

Fig. 5. Mean subchannel comparison between with reuse and without reuse of subchannels

The mean subchannel assignment per AP with min $\mathrm{RC}$ is shown in Fig. 7 where in Fig. 7(a) scaling value 1, and in Fig. 7(b) scaling value 2 are used. When scaling value 2 is used, the reuse 
of subchannels is increased and as a result the subchannels assignments in this case are higher than that of scaling value 1. In Fig. 7(a), as the traffic load of AP2 and AP4 are varying and reuse of subchannels is low, they get higher number of subchannels on increasing load whereby as the load of AP0, AP1 and AP3 are fixed, they get lower number of subchannels on increasing load. In Fig. 7(b), AP0, AP1, AP3 and AP4 reuse their subchannels. At low load situation, as AP2 has lower resource requirement, the remaining four APs get higher number of subchannels. When load of the AP2 increases, it gets higher number of subchannels; thereby, the assigned number of subchannels of AP0, AP1, AP3 and AP4 decreases. Here mainly, AP4 gets the higher number of orthogonal subchannels and the AP0, AP1 and AP3 reuse the subchannels of AP4. Due to reuse of subchannels, AP4 on average gets one subchannel more than that of AP2 in the case of high traffic load. Accordingly, when simulation is done with scaling value 3 , the mean delay decreases further which is shown in Fig. 6.

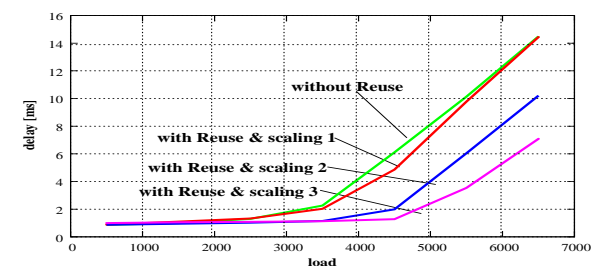

Fig. 6. Mean delay comparison between with reuse and without reuse of subchannels with different scaling value.

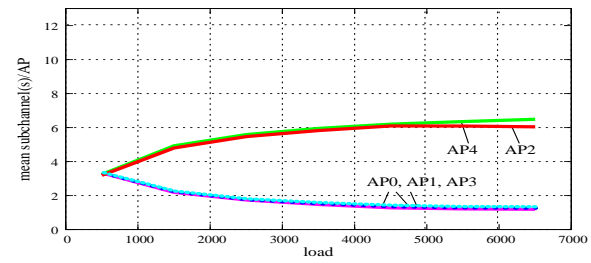

(a) Scaling value 1 .

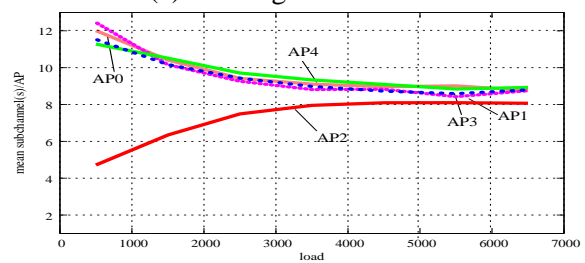

(b) Scaling value 2

Fig. 7. Mean Subcahnnels per AP

\section{CONCLUSION}

In this work the effect of interference aware frequency reuse is focused by using a hierarchical radio resource management (HRRM) scheme. The APC handles interference by exploiting the adaptive reuse partitioning. The simulation results using different scaling of distances between the APs show that the effect of frequency reuse greatly impacts the performance of OFDMA systems. By choosing the proper reuse constraint for a particular scenario, the packet losses can be reduced significantly.

As a future work, if the dynamic selection of the reuse constraints can be done by tracing the modulation scheme of the APs, this presented HRRM would be able to utilize radio resources in an optimized manner.

\section{ACKNOWLEDGEMENT}

We would like to thanks to Prof. B. Walke for his invaluable advice during the work and Dr. Nick Graham for reviewing the draft version of the paper and partial financial support.

\section{REFERENCES}

[1] S. Cheong, W. Cheng and K. Letaief, "Multiuser OFDM with Adaptive Subcarrier, Bit, and Power Allocation," IEEE Journal on Selected Areas in Communications, vol.7, pp.1747-1758, October 1999.

[2] T. Rappaport, Wireless Communications Principles and Practices, Prentice Hall PTR, United States of America, 2002.

[3] T. M. Cover and J.A. Thomas, Elements of Information Theory, Wiley, New York, 1991.

[4] H. FuruKawa and Y. Akaiwa, "Self Organized Reuse Partitioning, Dynamic Channel Assignment

Method in Cellular systems," Proceedings of the IEEE VTC, pp. 524-527, 1993.

[5] C. Bettstetter, "Mobility modeling in wireless networks: categorization, smooth movement, and border effects," ACM SIGMOBILE Mobile Computing and Communications Review, vol.5 no.3, pp.55-66, July 2001.

[6] W. Fischer, K. Meier-Hellstern,"The Markov Modulated Poisson Process (MMPP) cookbook", Performance Evaluation" vol.18 no.2, pp.149171, September 1993.

[7] NS-2: http://www.isi.edu/nsnam/ns/NS-2 .

[8] B. Roy, Dynamic Subchannel Allocation in a Multi-cellular OFDMA System based on Interference Measurement and Traffic Situation, Master Thesis, ComNets, RWTH Aachen University, December 2005.

[9] C.G. Org and F. Schreiber, "The RESTART/LRE Method for Rare Event Simulation," In Proceedings of the Winter Simulation Conference, pp. 390-400, Coronado, USA, 1996.

[10] H. Yin and H. Liu, "An Afficient Multiuser Loading Algorithm for OFDM-based Broadband Wireless Systems," IEEE Global Telecommunications Conference, vol.1, pp.103-107, November 2000. 